\title{
Transatlantica
}

Revue d'études américaines. American Studies Journal

$1 \mid 2015$

The Voting Rights Act at 50 / Hidden in Plain Sight:

Deep Time and American Literature

\section{Jonathan Pollock, Lire Les Cantos d'Ezra Pound}

\section{Charlotte Estrade}

\section{(2) OpenEdition \\ Journals}

Electronic version

URL: https://journals.openedition.org/transatlantica/7285

DOI: 10.4000/transatlantica.7285

ISSN: $1765-2766$

Publisher

Association française d'Etudes Américaines (AFEA)

\section{Electronic reference}

Charlotte Estrade, "Jonathan Pollock, Lire Les Cantos d'Ezra Pound", Transatlantica [Online], 1 | 2015 Online since 08 June 2015, connection on 04 February 2023. URL: http://journals.openedition.org/ transatlantica/7285 ; DOI: https://doi.org/10.4000/transatlantica.7285

This text was automatically generated on 4 February 2023

\section{(c) (i) () $\Theta$}

Creative Commons - Attribution-NonCommercial-NoDerivatives 4.0 International - CC BY-NC-ND 4.0 https://creativecommons.org/licenses/by-nc-nd/4.0/ 


\title{
Jonathan Pollock, Lire Les Cantos d'Ezra Pound
}

\author{
Charlotte Estrade
}

\section{REFERENCES}

POLLOCK, Jonathan, Lire Les Cantos d'Ezra Pound, Paris, Hermann Éditeurs, Collection « Fictions pensantes », 2014, 269 pages, ISBN 978270568906 3, 22 euros

1 «Les Cantos d'Ezra Pound sont [...] très peu lus, et pour cause : ils sont illisibles ». Tels sont les propos liminaires du chapitre XI de Lire Les Cantos d'Ezra Pound écrit par Jonathan Pollock. Dans cet ouvrage, l'auteur relève un défi multiple : celui de rendre accessible à un public francophone une œuvre difficile majeure de la poésie américaine, caractérisée par sa polyphonie, son plurilinguisme et son idiosyncrasie, auxquels s'ajoutent parfois la mauvaise réputation du poète notamment pour des raisons idéologiques, comme le rappelle l'auteur $(5,171)$. L'ouvrage comble un grand vide, puisqu'il n'y a pas, à ma connaissance, d'ouvrage en français sur les seuls cantos envisagés dans leur totalité. D'autre part, la réflexion philosophique stimulante apportée par l'ouvrage de Pollock est menée dans une langue élégante et claire, qui touche à des sujets très variés (et pas seulement à cause de son objet d'étude). Lire Les Cantos d'Ezra Pound, bien que dense, se lit avec grand plaisir.

2 L'ouvrage de Pollock est d'une qualité et d'une efficacité rares, sans jamais sacrifier la clarté de l'exposé. Il rebrasse bien sûr des thèmes essentiels soulevés par l'œuvre et attendus dans une telle analyse, tels la réécriture de l'histoire et la mémoire, l'économie, le modèle homérique, le vortex, ou encore l'emprunt et l'héritage, mais en donnant une impulsion nouvelle à la réflexion, et propose des outils intéressants et originaux pour la lecture et l'analyse.

3 Lire Les Cantos d'Ezra Pound se divise en douze chapitres (chacun est organisé autour de quelques points précis), encadrés par un «Prologue: Montagne ou vortex? » et un «Épilogue: La littoralité ». Le prologue définit tout d'abord les différents types de 
difficultés qui font que l'œuvre de Pound, rapprochée de celle d'Antonin Artaud, demeure très peu lue, pour la replacer dans un contexte bien particulier : celui, d'une part, du Vorticisme anglais qui fait du « choc des contraires » ainsi que de leur « mise en rapport " (21) son principe fondamental, et d'autre part celui des deux guerres mondiales.

4 La guerre constitue le point de départ du premier chapitre, intitulé «Bellum cano ", où Pollock analyse la préoccupation poundienne pour l'origine et les sources (et donc sa réécriture d'Homère, et en particulier de l'épisode de la descente aux Enfers), à l'aune d'une civilisation européenne (dans laquelle Pound vit) dont la destruction va paradoxalement amener un renouvellement poétique. Ce premier chapitre donne également des repères biographiques, tout en expliquant efficacement le cheminement intellectuel qui a amené Pound à défendre ardemment le fascisme.

5 «Une autre économie », le deuxième chapitre de l'ouvrage, expose avec une clarté et une objectivité exemplaires la pensée poundienne dans sa dimension politique, économique, et la façon dont Pound envisage ces problématiques comme liées à celle du langage (voir pages 54-55 en particulier), analysé et repensé grâce à l'étude des idéogrammes chinois notamment. Pollock permet ainsi (64-65) de démêler et clarifier des notions (cosmopolitisme, démocratie) qui déchaînent souvent les passions lorsqu'on les aborde en rapport avec le poète fasciste.

6 Le chapitre III (« Devenir personne ») commence par s'attaquer au problème épineux de la «structure » des Cantos, sur lequel l'ouvrage revient à plusieurs reprises sans pour autant se répéter - à cet égard, Pollock adopte de façon tout à fait bienvenue la structure en spirale qu'il dégage chez Pound, ce qui permet un ouvrage complet et sans les lourdeurs que pourraient provoquer un propos sur un sujet aussi complexe et encyclopédique. Pollock rappelle différentes façons de concevoir l'organisation du poème (prend-il pour modèle Dante? Homère? la fugue musicale? un principe thématique ?), pour lui préférer le terme tout à fait pertinent de «texture » (78) et insister sur le retour et la mémoire tels qu'ils sont véhiculés par la reprise de quelques épisodes homériques permettant à Pound d'écrire une œuvre protéiforme et ouverte qui brouille sans cesse la notion même d'auteur.

7 Le chapitre IV («Brisure et fusion») analyse plus en détail la loi ou la continuité qui guiderait l'écriture ou constituerait «l'architecture mobile des Cantos» (99). A l'agencement dicté par la très problématique «méthode idéogrammatique " proposée par Pound, mais qui montre vite ses limites, Pollock propose d'analyser les Cantos en termes de disjonction et de mobilité, ou de métamorphose, paradoxalement rendues par l'utilisation de la conjonction «et» (dont Pollock montrer qu'elle fait autant conjonction que disjonction) omniprésente dans le poème poundien et qui permet le mouvement, principe directeur déjà à l'œuvre dans les manifestes vorticistes. Comme dans d'autres chapitres, Pollock introduit ici des remarques bienvenues sur l'influence de Bergson, qui seront approfondies quelques chapitres plus loin.

8 «Turbulences et bacchanales » débute avec un détour par les sciences de la nature justifié par l'importance accordée par Pound au vortex -, et notamment une définition très technique du phénomène de convection (113-6), qui sert de modèle à l'analyse des " processus dramatiques et rhétoriques » dans Les Bacchantes d'Euripide et Les Cantos de Pound. Pollock explique ici Les Cantos en termes de flux et de contingences réagencés par le poète, et propose de façon convaincante «la notion de 'morcelage réenchaîné'« plutôt que la juxtaposition comme méthode de composition des Cantos. 
9 Le chapitre VI («La part du hasard») approfondit la notion de vortex dans son sens mathématique et philosophique. A la lumière de la philosophie épicurienne (notamment véhiculée par Lucrèce) et des théories de Louis Althusser à qui Pollock emprunte le concept de rencontre, l'auteur dissèque la conception poundienne de la nécessité, de l'aléatoire et du contingent dans les événements des Cantos.

Ce n'est qu'au chapitre suivant («Le vertige de la mémoire ») que Pollock rappelle la définition poundienne du vortex, pour préciser le type de mémoire à l'œuvre dans Les Cantos, qui relèvent plus, pour Pollock, de la "remémoration » que de "l'inscription » (153) en dépit de leur but avoué de constituer «l'histoire de la tribu». Cette remémoration est expliquée par des références à Bergson dont les définitions du flux et de la durée ont marqué l'époque moderniste plus généralement. Pollock relit Pound à l'aide de l'image du cône bergsonien qui permet de différencier différents types de mémoire et une perception du temps bien particulière dans l'œuvre poétique de Pound. Si de nombreux liens sont dégagés entre Ezra Pound et William Butler Yeats au cours de l'ouvrage, on s'étonne qu'aucune mention ne soit faite des "gyres » yeatsiens, dont la dimension mémorielle, historique et ésotérique est comparable au cône bergsonien dans sa réactivation poundienne. Pollock clôt le chapitre VII sur l'idée que la contraction à l'œuvre dans les «images-souvenir " (165) présentes dans Les Cantos affecte aussi le langage. Exemple ultime de cette "contraction» du langage : le mot provençal noigandres qui résiste aux plus grands philologues interrogés par Pound et qui devient source poétique dans l'œuvre.

11 Le terme résiste aux contemporains universitaires de Pound, mais pas à Pollock, qui offre dans le chapitre VIII (« Noigandres») une interprétation convaincante, avec un détour bienvenu et stimulant par la réception et l'héritage brésiliens (la revue et le groupe Noigandres qui s'inscrivent dans la tradition poundienne et réactivent la « résistance », 172, à l'œuvre dans Les Cantos). Pollock propose dans ce chapitre une micro-lecture du Canto XX qui permet de montrer en quoi l'élucidation de l'encyclopédisme des Cantos ne résout pas le problème de la lecture transformée par le «[m]ontage, et non pas collage » du texte poundien dont l'unité, l'ensemble, la totalité fait problème.

12 Dans le poétique chapitre IX intitulé «Renaissances », Pollock explore la philosophie chinoise et confucéenne telle qu'elle est reprise chez Pound, pour montrer comment les phénomènes cosmiques permettent également au poète de proposer une éthique dans un texte poétique marqué par la musique. Pollock montre comment nature, mythologie et rythmes sont interdépendants dans la poésie poundienne.

13 «La voie de Confucius » poursuit l'étude du penseur chinois qui a marqué Pound et insiste sur l'altérité qui caractérise cette pensée, ainsi que le caractère chinois idéogrammatique auquel Pound l'associe. Pollock revient sur les origines (notamment le rôle divinatoire) des idéogrammes ainsi que sur le type d'écriture qui en découle et la conception du langage qu'ils présupposent.

Le très beau chapitre XI sur "Le vertige du silence» commence par questionner la notion de «lecture » telle qu'on peut la penser en lien avec Les Cantos qui exigent du lecteur un autre type de lecture visant non la compréhension d'un tout structuré mais la «surpréhension" (232), terme que Pollock emprunte à Bernard Stiegler. L'expérience d'une langue poundienne singulière se fait aussi à travers le silence grandissant à mesure que l'on avance dans Les Cantos. Pollock différencie différents types de silences qui s'expliquent par plusieurs éléments (biographiques, poétique, etc.) 
et notamment l'utilisation des idéogrammes chinois dont la philosophie est bien expliquée et la dimension poétique tout à fait transmise. Le seul regret, dans ce chapitre XI où Pollock indique pourtant la dimension visuelle des Cantos (234) et décrit des idéogrammes chinois ainsi que leur disposition sur la page du texte poétique (243), est de n'en voir précisément aucun, alors même que Pollock indique les rapports de Pound au Vorticisme et mentionne l'entourage artistique de Pound (7). De même, ailleurs dans l'ouvrage de Pollock, tous les extraits cités par Pound en alphabet grec dans son long poème sont repris par Pollock en translittération dans l'alphabet latin et mis entre crochets. Certes, cela permet à tout lecteur de « lire », de vocaliser le texte et Pollock envisage surtout un "lecteur-auditeur" (181) - mais dans plusieurs exemples $(91,227)$ et comme souvent chez Pound, le texte grec est redondant avec le vers poétique qui le suit immédiatement. Il faudra se contenter de descriptions pour tous les caractères hors alphabet latin: pictogrammes, hiéroglyphes, "notation en neumes » (252), et/ou avoir les Cantos à portée de main pour la dimension visuelle du poème. Malgré cela, les citations proposées par Pollock sont généreuses et toutes traduites en français.

Le dernier chapitre ( $X I I$. Le vol de l'alouette») aborde la question du paradis, inséparable chez Pound de plusieurs reprises littéraires et figurée poétiquement par la figure de l'alouette, dont l'analyse par Pollock donne lieu à de très belles pages.

L'épilogue, tout en rebrassant les idées essentielles de l'ouvrage, propose finalement comme guide de lecture non pas la métaphore qui fige du texte tissé, mais un refus de totalisation, une approche ouverte à la dialectique de l'œuvre complexe que sont Les Cantos, où la lecture serait navigation.

En somme, Lire Les Cantos d'Ezra Pound est un ouvrage dense, clair et stimulant. Les thèmes et problématiques essentiels sont bien amenés en début d'ouvrage; Pollock reprend et étoffe ensuite l'analyse. Le lecteur est ainsi guidé tant pour saisir l'économie et le but général du poème que dans l'appréciation de passages poétiques auxquels des micro-lectures détaillées et pertinentes viennent rendre justice (comme par exemple la belle analyse à la fois sémantique et grammaticale des pages 88-89). Les enjeux essentiels sont souvent synthétisés dans des formulations à la fois élégantes et efficaces qui offrent une lecture agréable et profitable tant aux spécialistes de poésie anglophone qu'à un lectorat francophone plus général ayant un intérêt pour les questions philosophiques en littérature notamment.

\section{AUTHORS}

\section{CHARLOTTE ESTRADE}

Université de Paris Ouest Nanterre - La Défense 